\title{
Non Destructive Evaluation of Damage and Failure of Fibre Reinforced Polymer Composites Using Ultrasonic Waves and Acoustic Emission
}

R. El Guerjouma, J. C. Baboux, D. Ducret, N. Godin, Ph. Guy, S. Huguet, Y. Jayet; T. Monnier.

GEMPPM, UMR CNRS 5510, INSA de Lyon,

69621 Villeurbanne

\section{Introduction}

Applications of reinforced composites and heterogeneous solids are widespread, spanning technological areas of various aerospace and mechanical industries. A real challenge concerning these materials is their life time prediction when subjected to wide variety of environmental and mechanical loading conditions that can initiate damage and lead to failure. Indeed, damage at the smallest scales drives damage accumulation at larger length scales until some critical local damage state is attained that causes macroscopic failure. A key issue in predicting life time is to characterise distributed volumic and localised damage and to understand the mechanisms of its initiation, evolution and criticality and so, the identification of relevant precursors of failure. To answer to these questions, volumic and guided ultrasonic waves and acoustic emission are of particular interest ${ }^{[1]}$. As a matter of fact volumic ultrasonic wave propagation is sensitive to homogeneously distributed microcracks and represents in that case a good damage indicator ${ }^{[2,3]}$. Guided waves as Lamb waves especially when generated from inside the material using an inserted piezoelectric element offer a specific sensitivity to localised damage as crack or delamination ${ }^{[4,5]}$. Besides, acoustic emission which corresponds to the energy generated by the material during the damage processes is directly related to the damage mechanisms ${ }^{[6]}$ and so can give pertinent information about the damage initiation and development. In this paper, our aim is to show in the one hand the ability of volumic ultrasonic waves to characterise volumic damage of glass epoxy composites under hygrothermal ageing and also the ability of Lamb waves to detect and identify localised damage. In the other hand our purpose is to demonstrate the potentiality of acoustic emission in understanding the damage mechanisms that occurs during a tensile test of polymer fibre composites and to discriminate in real time the different types of damage occurring at the microscopic scale. 
The first part of this paper deals with the ultrasonic characterisation of the hygrothermal aging of a glass fibre reinforced epoxy matrix composite. The characterisation of this damage is performed using an immersion technique through its effects on longitudinal and shear ultrasonic velocities measured as function of the propagation direction. The anisotropic elastic constants of the material are recovered from the ultrasonic velocities using an optimization process. Anisotropic damage is assessed in terms of loss of stiffness and damage parameters. A microscopic investigation conducted using scanning electronic microscopy (SEM) on the damaged material confirms the anisotropy of the damage.

This approach applies to a homogeneously distributed damage, but monotoring the health of a structure depends also on the presence of localised defects such as cracks or delaminations.

So in the second part of this paper, an ultrasonic health monitoring technique allowing to recover both kind of information is presented. This method consists in inserting a piezoelectric element within the structure. The element dimensions are designed in order to uncouple the frequency ranges of the thickness and radial vibration modes. The thickness mode is used to monitor the homogeneous ageing of the structure through electrical impedance measurement ${ }^{[4,5]}$ while the radial vibration mode, is used to generate and detect Lamb waves, which have the advantage of propagating over long distances and offering specific sensitivity of various modes to different kinds of defects. The ability of the proposed technique to detect localised macroscopic damage resulting from low speed impact in carbon epoxy composite.

The third part of the paper deals with acoustic emission (AE), this technique has been used to characterise damage evolution in a glass fibre/polyester composite. We worked on different types of samples that we expected to produce preferential damage mechanisms. AE was first studied via the parameters and waveforms of the signals. Two main types of signals were identified, coming from the two awaited damage mechanisms : matrix cracking and fibre/matrix decohesions. But the difficulty to separate objectively two clusters within a large quantity of signal leads us to use a neural networks based pattern recognition techniques. This approach is well adapted to processes involving many variables. So, the combination of acoustic emission amplitude distributions and neural networks, in the form of a Kohonen selforganising map ${ }^{[7]}$ was successfully employed to discriminate signals originating from different damage types. As a conclusion we discuss the possibility of predicting the failure of unidirectional composites from AE signals when the material is subjected to a tensile test. 


\section{Ultrasonic Evaluation of hygrothermal aging.}

\subsection{Material and aging conditions}

The material under investigation is E glass fiber reinforced epoxy matrix manufactured in the form of thin plates ( $2 \mathrm{~mm}$ thickness). The cure cycle was $2 \mathrm{~h}$ at $125{ }^{\circ} \mathrm{C}$ under 3 bars of pressure. The volume fraction of fibre was $68 \%$. The sample $(5 \times 5 \mathrm{~cm})$ was placed in a container of distilled water at $70{ }^{\circ} \mathrm{C}$ and was removed at 10, 20 and 25 days for velocity measurements.

\section{2 Volumic Ultrasonic Evaluation : Elastic constants determination}

The ultrasonic evaluation of elastic properties of materials is based on the relationship between the elastic constants of material and the phase velocities of volumic ultrasonic waves. Considering progressive plane waves, the resolution of the wave propagation equation, for a homogeneous elastic and anisotropic media, gives the so-called Christoffel equation ${ }^{[4]}$. Ultrasonic evaluation consists in recovering the elastic constants from ultrasonic velocity measurements. This is typically an inverse problem. Concerning unidirectional composites, transverse isotropy with five independent elastic constants is generally considered. However, even for such composites, orthotropy may appear due to particular arrangement of the fibers or to matrix texture in the initial state or to microstructural changes during damage development $^{[4][5][6]}$.

Fig. 1. Orthotropic elastic matrix, symmetry axes of the material and planes of propagation

To solve the aforementioned inverse problem using a unique sample in the form of plate with parallel faces an optimisation process has been performed ${ }^{[8,9]}$. It is based on multiple velocity measurements in various planes of propagation (symmetry and non-symmetry planes). Such measurements are generally obtained by using an immersion technique, varying the angle of incidence. The problem is mostly overprescripted, there being in general more data (measured velocities) than unknown parameters (elastic constants) to determine. The most suitable method to solve this problem is to use an optimisation process based on a non-linear minimization technique. In our case, elastic constants determination is performed by minimising the sum of the squares of the deviation between experimental and theoretical 
velocities. The minimization algorithm used is the Levenberg-Marquardt one ${ }^{[10]}$. For an orthotropic material there are three symmetry planes and nine independent elastic constants. For unidirectionnal composites these planes are typically planes P13, P23 and P12 as shown in Figure 1 which also represents the nine independent elastic constants of the material. In our case, because of the low thickness of the material, seven of the nine independent elastic constants $\left(\mathrm{C}_{11}, \mathrm{C}_{22}, \mathrm{C}_{33}, \mathrm{C}_{44}, \mathrm{C}_{55}, \mathrm{C}_{13}, \mathrm{C}_{23}\right)$ were determined from quasi-longitudinal (QL) and quasi-shear wave velocities measurements in two planes of symmetry namely P13 and P23 and in a non principal plane which direction makes an angle of $45^{\circ}$ against the symmetry direction of the material (plane P45 in Figure 1).

\subsection{Damage characterisation}

The aforementioned planes of propagation, the ultrasonic velocities versus direction of propagation are measured for the initial state and after 10, 20 and 25 days aging. The ultrasonic wave velocities were found to decrease as the aging time increases. In addition to the reduction in velocities, the angular dependence of the velocity is changed due to the evolution of the aging damage especially in the P23 plane (perpendicular to the fibre direction).

From these velocity measurements, the elastic constants of the material assumed orthotropic, are determined as a function of hygrothermal aging. Table 1 shows these elastic constants at different stages of hygrothermal aging. One can notice that in the undamaged state the composite can be assumed to exhibits transverse isotropy. As the hygrothemal aging progresses all elastic constants decrease. In the plane P23 $\left(\mathrm{C}_{22}, \mathrm{C}_{33}, \mathrm{C}_{23}, \mathrm{C}_{44}\right)$ the loss of stiffness is more important than in plane $\mathrm{P} 13\left(\mathrm{C}_{11}, \mathrm{C}_{33}, \mathrm{C}_{13}, \mathrm{C}_{55}\right)$.

Table 1. Elastic constants (in GPa) for different aging states

The anisotropic degradation of the elastic constants with hygrothermal aging indicates anisotropic microstructural changes within the material. So, ultrasonic velocities and furthermore, the elastic constants appear to be good damage indicators. In this case, the collective effect of the homogeneously distributed microcracks has a significant influence on the macroscopic behaviour at the wavelength scale in the sense of continuum mechanics. So damage can be treated in the framework of continuum damage mechanics (CDM). A characteristic of CDM is the introduction of an internal damage variable into constitutive 
equations. This variable takes into account, on the macroscopic scale, the microscopic deterioration of the material ${ }^{[3]}$. So, the consideration of irreversible relative variations of the stiffness constants $\mathrm{C}_{\mathrm{ij}}$ from the initial state $\mathrm{C}_{\mathrm{ij}}{ }^{0}$ leads to a symmetrical fourth order damage tensor $\mathbf{D}$ which diagonal components $\mathrm{D}_{\mathrm{ii}}$ are given by the relation :

$\mathrm{D}_{\mathrm{ii}}=1-\mathrm{C}_{\mathrm{ii}} / \mathrm{C}_{\mathrm{ii}}{ }^{0}$

The Dii parameters are related to principal directions and principal planes. They vary from 0 (undamaged state) to 1 (failure of the volume element scale)

In our case, all measured diagonal internal damage variables $D_{i i}$ increase with the hygrothermal ageing but these variations are different according to the considered directions and planes as shown in Figure 2 (direction 1 is the fibre direction, 2 the transverse direction and 3 the thickness direction).

\section{Fig. 2. Damage evolution}

This fact clearly demonstrates the anisotropy of the damage. The greatest increase occurs for the shear damage in the plane P23, where the term D44 (related to $\mathrm{C}_{44}$ or $\mathrm{G}_{23}$ ) reaches 0.45 . The less important ones are along 1 and 2 axes and concerns the elastic constants $C_{11}$ and $C_{22}$. We note also an important increase of $\mathrm{D}_{33}$ related to $\mathrm{C}_{33}$ showing an important damage through the thickness. These macroscopic effects of damage result from oriented micro-cracks within the material. The large increase of $\mathrm{D}_{44}$ results from the damage of the fibre/matrix interface.

To clear this point, a microscopic investigation using scanning electronic microscope (SEM) on the damaged sample was performed. The SEM analysis shows an important debonding at the fiber-matrix interface mostly located at the pole of the fibres in the thickness direction as shown in Figure 3. The observed damage is consistent with the ultrasonic results.

Fig. 3: SEM observation : fiber-matrix debonding

\section{Damage detection through Lamb waves in large composite structures.}

As stated before, the health of a structure depends on the homogeneously distributed degradation of its mechanical properties during its life cycle as well as on the presence of 
localised defects such as cracks or delaminations. The present ultrasonic health monitoring technique allows to recover both kinds of information. The insertion of a piezoelectric element in the composite structure avoids traditional techniques limitations, such as coupling

reproducibility $[4,5,11]$. The element dimensions are designed in order to uncouple the frequency ranges of the thickness and radial vibration modes. The embedded sensor is operated in the frequency range of its radial vibration mode, enabling the excitation of guided waves. A particular Lamb mode is selected by tuning the driving frequency, in relation with the radial dimensions of the sensor ${ }^{[11]}$. The velocity and the induced stress and strain distributions in the through-thickness direction vary with frequency, so each Lamb mode presents a specific sensitivity to a given kind of defect ${ }^{[12,13]}$.

\subsection{Experimental conditions}

The described experiments have been carried out upon a $6 \mathrm{~mm}$-thick Carbon Fibres Reinforced Plastic (CFRP) plate of $255 \times 300 \mathrm{~mm}^{2}$ and made-up of 22 carbon/epoxy unidirectional plies stacked according to the sequence $\left[45^{\circ},\left[135^{\circ}, 45^{\circ}{ }_{2}\right]_{10}, 135^{\circ}\right]$ where $0^{\circ}$ and $90^{\circ}$ denotes the directions of the plate edges.

For practical considerations, each sample was instrumented with two identical sensors bonded on the surface instead of being embedded into the structure during elaboration. In the literature, it is widely agreed that low-speed impacts produce matrix cracks, fibre breakage and delamination. The response of the structure to an impact is linked to many parameters, such as the mechanical properties of the composite material, the plate thickness or the stacking sequence of the plies. Then realistic damages such as those observed during the service of real aeronautic or automotive structures, were induced by means of low speed impacts (velocity below 20-40 m. s ${ }^{-1}$ for that particular kind of structure) ${ }^{[14]}$.

We used a calibrated impacting device, with a $20-\mathrm{mm}$ in diameter hemispherical impactor, allowing the damaging threshold below which no critical damage is induced to be easily determined between 6.13 and 6.38 joules. Then four successive impacts of energy 2, 4, 8 and 8 joules were performed at the same location, in the path between the emitter and the receiver, this path being parallel to the first ply fibres direction.

The existence of an actual damage has been checked through a classical C-scan procedure. This showed that the delamination occurs at the interface between plies having different fibre orientations rather than between plies with the same one. Moreover, these delaminations exhibit a classical 2-lobe pattern, the principal axis of which having the lower ply fibre 
direction. Each pattern is then orthogonal to the previous one and the deeper the delamination is, the larger it is because of the greater bending of the sample at this depth during the impact.

\subsection{Use of Lamb waveforms for impact induced damage detection}

The emitter is driven with a 5-cycles toneburst of adjustable frequency. The transmitted waveforms are recorded at the receiver. Figure 4 shows the $S_{0}$ transmitted waveforms in the sane case and after the damaging process. Waveforms corresponding to the first two impacts were found to be very close to the reference waveform, so they are not represented here. The $\mathrm{S}_{0}$ mode was chosen, as it appeared to give better results than the first anti-symmetrical Lamb mode $A_{0}$ for the detection of this kind of «in-plane» flaw. As it can be seen in Figure 4, the time signals are different but it is difficult to define a discriminating parameter.

A set of one hundred raw reference signatures was recorded in the sane plate, and ten raw signatures were recorded after each impact. This kind of statistical analysis takes into account the dispersion of the results. In Figure 5, the maximum magnitudes of the transmitted waveform spectra are plotted in the complex plane. The grid is made of circular curves representing several attenuation levels $(0 \mathrm{~dB}$ i.e. reference level corresponds to the average amplitude of the reference measurements), and radial straight lines, which angular separation corresponds to the phase shift induced by a time delay equal to one sampling point. This 2-D representation has the advantage of combining the information from two significant parameters: the transmitted spectrum maximum magnitude and its associated phase. As illustrated in Figure 5 the different kinds of signatures are distinctly clustered, enabling a better discrimination between the non-faulted condition and the damage signatures. This has been observed in many of the cases we examined. It is not possible to distinguish the sets of points representing the first two impacts from the references, but this result is consistent with the fact that their energy was below the damaging threshold. High-energy impacts are easily detected as the overall amplitude of the set of stars representing the first 8-joules impact is attenuated more than $1 \mathrm{~dB}$ and its phase is shifted from the set of references. Moreover, one can easily notice that a second heavy impact at the same location and of the same energy continues to damage the composite, as the overall amplitude of the second 8-joules impact signatures is more attenuated and also more phase shifted.

\subsection{DISCUSSION}


This study has shown the efficiency of the attached piezoelectric element to generate and detect Lamb modes. The interest of such a multi-modal technique has been pointed out, since according to its sensitivity to a given defect in a given structure a particular Lamb mode can be arbitrary selected by tuning the excitation frequency on the bonded sensor. For the specific case of the structures described here, we observed the evolution of the maximum spectral amplitude and its associated phase, as these parameters of the propagating modes were previously defined as mainly significant ${ }^{[5]}$.

These results have demonstrated that the $S_{0}$ mode was particularly sensitive for the detection of the delaminations in CFRP plates. In order to interpret the interaction of a given Lamb mode with such realistic damage, we have started a theoretical study combining finite element method (FEM) and analytical approach.

\section{Unsupervised clustering techniques for the characterisation of failure modes in a Glass Fiber reinforced composite by acoustic emission}

\section{1 Experimental results and conventional analysis of acoustic emission signals}

Acoustic emission (AE) has been developed over the last two decades as a non destructive evaluation technique and as an useful tool for material research ${ }^{[6]}$. AE is an efficient method to monitor, in real time, damage growth in both structural components and laboratory specimens ${ }^{[15,16]}$. In loaded materials, the strain-energy release due to microstructural changes results in stress-wave propagation. In the case of composite materials, many mechanisms have been confirmed as $\mathrm{AE}{ }^{[17,18,19]}$ sources including matrix cracking, fibre-matrix interface debonding, fibre fracture and delamination..

In this work, acoustic Emission (AE) was used to discriminate in real time the different types of damage occurring in a fribrous composite under mechanical load. For that purpose, we worked on polyester and glass/polyester unidirectional specimens, subjected to tensile loading within different configurations, awaiting preferential damage modes in the material. Three types of samples were used in this study : pure resin, $45^{\circ}$ and $90^{\circ}$ off-axis unidirectional samples. Each of these samples were chosen to generate preferentially particular damage modes during tests : matrix fracture for resin samples, mainly matrix fracture with some decohesions for $90^{\circ}$ off-axis, mainly decohesion with some matrix fracture for $45^{\circ}$ off-axis. 
Acoustic emission measurements were achieved by the use of two resonant micro80 PAC sensors (Figure 6), applied on the faces of the samples during testing. The composite used was made of E-type Vetrotex glass fibres and Scott Bader polyester matrix with Peroximon K1 hardener. Polymerisation was achieved at room temperature during approximately 12 hours. Unidirectional laminates were made of 12 prepregs and were about $2.5 \mathrm{~mm}$ thick with $75 \%$ of fibres in weight.

Tensile tests on pure resin samples generated few AE activity. All AE waves detected can be considered as burst type signal which implies that the AE waves were generated during dynamic and discontinuous microfracture process in the sample. Signals appearing during the main part of the tests, were associated to the nucleation and growth of vacuoles inside the resin, in other words, matrix microcrack formation. From the test on resin samples, the acoustic signature of matrix fracture was determined : slowly rising waveform and low amplitude.

The amplitudes of the collected signals recorded during tests on unidirectional samples loaded in the transverse direction to fibres $\left(90^{\circ}\right.$ off-axis) are mainly distributed in two zones exhibiting a bimodal behaviour (figure 7). About $70 \%$ of them have amplitudes in the range of 50 to $70 \mathrm{~dB}$, with waveforms similar to those observed in the tests on pure resin ; we will refer these signals as "A-type" (Figure 8, lower left). The $30 \%$ left signals have amplitudes in the range of 70 to $90 \mathrm{~dB}$ and waveforms quite different from the waveforms of A-type signals, with shorter decay time and higher energies. These signals will be referred as "B-type" (Figure 8, lower right). The definition of the signal amplitude ranges are somewhat arbitrary. There was overlap in the parameter data for various signals. Nevertheless, such simple arbitrary guidelines can appear to be useful.

The similarities in waveforms found between A-type signals in $90^{\circ}$ off-axis tests and signals from pure resin tests let us think that the source mechanism is the same in both cases, i.e. matrix fracture. In agreement with the fact that mode I cracking is the main fracture mode for this kind of sample. B-type signals (of amplitudes between 70 and $90 \mathrm{~dB}$ ) are detected only during the second half of the test as can be seen in figure 7, whereas the activity of A-type seems quite continuous. So, A-type and B-type seem to correspond to different mechanisms inside the material. B-type though must have another mechanical origin, occurring after certain matrix damage level has been attained.

Figure 8 represents, for the tests on samples in the $45^{\circ}$ direction to fibres, the amplitude distribution and typical waveforms. A-type and B-type signals can be observed in the same amplitude zones as previously and with very close parameters and waveforms. However, B- 
type signals are found much more numerous than A-type in these tests (80\%). The main expected damage mode in these types of tests, considering that $45^{\circ}$ is the direction in which shear stress is maximum along the direction of fibres ${ }^{[20]}$, is fibre/matrix decohesion. So the source of B-type signals, predominant in this configuration, corresponds to decohesion. This is also consistent with their presence in the $90^{\circ}$ tests, appearing chronologically after A-type, when matrix fracture surrounds the fibres. The fact that the fibre/matrix decohesions are dominant in the $45^{\circ}$ tests was confirmed by SEM (Scanning Electronic Microscopy) observations.

\subsection{Statistical analysis : Kohonen self-organising feature map}

In order to separate numerically the two types of signals that we observed previously, a neural network approach was implemented. Such techniques have demonstrated their capabilities in functionally modelling processes involving many variables ${ }^{[21]}$. More precisely, a Kohonen self-organising feature map ${ }^{[19]}$ was used to organise feature vectors into clusters so that points within one cluster are more similar to each others than vectors belonging to other clusters. The inputs were six AE features taken from the signals. The network response clearly exhibits two main clusters (figure 10), corresponding to the two types of signals previously observed. The Kohonen map could then be used to compute the chronology of appearance of A-type or B-type signals during tests, as an indicator of the damaging kinetics of each damaging mechanisms. Interpretation of clustering results on a macroscopic level is performed by plotting the cumulative events of each class versus load. An example is given in Figure 10 for two tests at $90^{\circ}$ and $45^{\circ}$ from fibres. It indicates a typical evolution of AE event, for the discriminated source mechanisms. For the $90^{\circ}$ sample it is observed that the two classes exhibit similar activity trends. The fraction of A-type signals for the $90^{\circ}$ sample evolved more prominently than for the $45^{\circ}$ sample, from the beginning to the end of deformation. On the contrary, a larger fraction of B-type signal was detected for the $45^{\circ}$ than for which. decohesion is the dominant damage mechanism. Let us also note that the number of B-type signals associated with decohesion, greatly increases at the end of tests, causing the final failure of the material.

\section{Concluding remarks : Non Destructive Evaluation and failure Prediction}


Predicting damage evolution up to and including final failure is becoming a crucial and central question in modern mechanics for composite materials and structures. To answear to such a question concerning fibrous composites, a mechanics approach consist of using cumulative damage models in which the heterogeneous medium is modelled as an effective medium characterised by average properties, including an increasing global damage parameter ${ }^{[22,23]}$. In this field, it is clear that ultrasonic waves can bring pertinent informations especially for the evaluation of anisotropic damage and for the validation of CDM models. However if CDM based models associated to ultrasonic evaluation are very suitable for damage assessment and evolution, they are generally not able to predict the final failure. Failure mostly appears to be a critical point especially when it is not controlled by a single event accumulation (nucleation and growth of microcracks) but rather by multiple events sucession that lead to the catastrophic growth of a dominant crack.

From this point of view failure can be seen as a co-operative phenomena. Indeed, in the case of complex systems recent works using statistical physics approaches have shown that in somme cases, these statistical models successfully predict failure ${ }^{[24]}$. Some authors suggested ${ }^{[25]}$ that rupture of sufficiently heterogeneous media should exhibit some universal behaviour. In this field, recent works ${ }^{[26,27]}$ have shown that $\mathrm{AE}$ has a great potentiality. It has been observed that the elastic energy released per unit time, on average increases as a power law of the time-to-failure ${ }^{[26,27]}$. The rupture was found to occur as the culmination of the progressive nucleation, growth and fusion between microcraks.

In this paper, we have shown that $\mathrm{AE}$ can help in identifying the different damage mechanisms that occurs in a fibrous composite subjected to a tensile test. As a matter of fact AE signals produced during damage creation and development contain relevant precursory information about damage that can be used in a failure prediction scheme. So, the statistical properties and the location of fracture precursors in heterogeneous materials could be studied by recording their acoustic emission as a function of an extern parameter (time, applied load, temperature.....). The general method for developing a prediction model is to first determine which detected AE are relevant precursory phenomena and then develop an statistical model which allows one to relate these precursors to the final failure. In our case, the AE damage mechanism identification method we have presented in this study appear to be an efficient tool to select the most pertinent precursor to failure for a life time prediction approach. Another important question that needs to be addressed to guide prediction concerns the influence of the size of the structure and the type of loading on the existence of a power law. The problem of failure and life time prediction is rather complicated and need to be treated as 
a multidisciplinary approach involving ultrasonics, mechanics of material, statistic physics and material sciences engeneering. In the paper we have shown that ultrasonic propagation and acoustic emission can bring a lot in the understanding of the damage mechanisms of fibrous composite materials. These results are very encouraging and the next step of our study concerns now the failure and life time prediction of these materials.

\section{REFERENCES}

[1] Royer D. and Dieulesaint E. «Ondes élastiques dans les solides - Tome 1 : Propagation libre et guidée. » Paris : Masson, 1996. 328 p.

[2] Rokhlin S.I., Chu Y.C. and Huang W. Mechanics of materials, 1995, Vol. 21, N 1, p251263

[3] Baste S., El Guerjouma R. and Audoin B. Mechanics of materials, 1992, Vol. 14, N 1, p. $15-31$

[4] Perrissin-Fabert, Y. Jayet, Ultrasonics, Vol. 32, $\mathrm{N}^{\circ}$ 2, pp. 107-112, 1994

[5] Monnier, Y. Jayet, P. Guy, and J.C. Baboux, Review of Progress in QNDE, Vol. 19, eds. D.O. Thompson and D.E. Chimenti, Plenum, New York, 2000

[6] R. K. Miller, Nondestructive Testing Hanbook, vol. 5, P. mcIntire editors, ASNT, 1987.

[7] Kohonen T., 1982, Biological Cybernetics, 44, pp. 135-140.

[8] Ducret D., El Guerjouma R., Guy P., M. R’Mili, Baboux JC, Merle P.. 'Composites Part A : applied Sciences and Manufacturing, 1999, Vol 31, $\mathrm{n}^{\circ} 1, \mathrm{pp} 45-55$

[9] Aristegui C. and Baste S. J. Acoust. Soc. Am., 1997, Vol. 101, № 2, p. 813-833.

[10] Press W.H., Teukolsy S.A., Vertterling W.T. and Flannery B.P. Numerical recipes in C. $2^{\text {nd }}$ edition. New York : Cambridge University Press, 1992.

[11] N. Saint-Pierre, Y. Jayet, P Guy, J. C. Baboux, Ultrasonics, Vol. 36, pp. 783-788, 1998

[12] N. Guo, P. Cawley, J. Acoust. Soc. Am., vol. 94, nº4, pp. 2240-2246, 1993

[13] K. Maslov, T. Kundu, Ultrasonics, vol. 35, pp. 141-150, 1997

[14] H. Berthelot, PhD Thesis, Ecole Centrale de Lyon, 1997

[15] N. Godin, M. R'Mili, P. Merle et J. C. Baboux, Comptes Rendus des $11^{e}$ Journées Nationales sur les Composites (JNC11), Arcachon, 18-20 Novembre 1998.

[16] M. Jacquesson, P. Paulmier, N. Godin, R. Fougères, ICCM-12, Paris 5-9 July 1999 p. 452.

[17] Barré, S., Benzeggagh, M.-L., Comp. Science Tech.; 52, pp. 369-376, 1994.

[18] Ely T. M., Hill E. K, Materials Evaluation, pp.288-294, 1995. 
[19] S. Huguet, N. Godin, R. Gaertner, L. Salmon, D. Villard, ECCM-9, Brighton UK, 4-7 Juin 2000.

[20] Gay, D. (1991) Matériaux composites (3e ed.) Hermes, Paris

[21] M. E. Fisher, E. V. K. Hill, Materials Evaluation, pp 1395, 1998

[22] P. Ladeveze, Computational Mechanics'1995, 17, pp 142-150

[23] Z. Xia, W.A. Curtin, P.W.M. Peters ‘Acta Materiala, 49, 2001, pp 273-287

[24] D. Sornette and C. vanneste, Phys. Rev. Lett. 68, pp.612, 1992.

[25] L. De Arcangelis, S. Redner, H. J. Herrmann, Journal de Physique Lettres 46, L585590,1985 .

[26] A. Guarino, A. Garcimartín and S. Ciliberto, European Journal of Physics B, vol. 6, pp. 13-24, 1998.

[27] J. C. Anifrani, C. Le Floc'h, D. Sornette and B. Souillard, J. Phys. I France 5, pp. 631638, 1995. 
$\left[\mathrm{C}_{\mathrm{IJ}}\right]=\left(\begin{array}{cccccc}\mathrm{C}_{11} & \mathrm{C}_{12} & \mathrm{C}_{13} & 0 & 0 & 0 \\ & \mathrm{C}_{22} & \mathrm{C}_{23} & 0 & 0 & 0 \\ & & \mathrm{C}_{33} & 0 & 0 & 0 \\ & & & \mathrm{C}_{44} & 0 & 0 \\ & \text { Sym. } & & & \mathrm{C}_{55} & 0 \\ & & & & & \mathrm{C}_{66}\end{array}\right)$

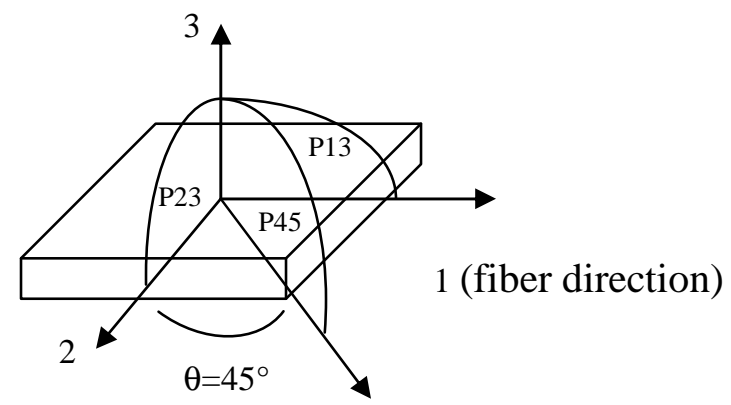

Figure 1. Orthotropic elastic matrix, symmetry axes of the material and planes of propagation

\begin{tabular}{|c|c|c|c|c|c|c|c|}
\hline Time(days) & $\mathrm{C}_{11}$ & $\mathrm{C}_{22}$ & $\mathrm{C}_{33}$ & $\mathrm{C}_{23}$ & $\mathrm{C}_{13}$ & $\mathrm{C}_{44}$ & $\mathrm{C}_{55}$ \\
\hline 0 & 71,5 & 30,2 & 30,7 & 13,2 & 15,4 & 7,7 & 8,3 \\
10 & 68,9 & 29,1 & 29,2 & 14,0 & 14,3 & 6,7 & 7,6 \\
20 & 63,1 & 26,5 & 22,3 & 10,8 & 10,4 & 4,8 & 6,7 \\
25 & 62,6 & 26,4 & 21,3 & 10,9 & 10,2 & 4,3 & 6,0 \\
\hline
\end{tabular}

Table I. Elastic constants (in GPa) for different aging states
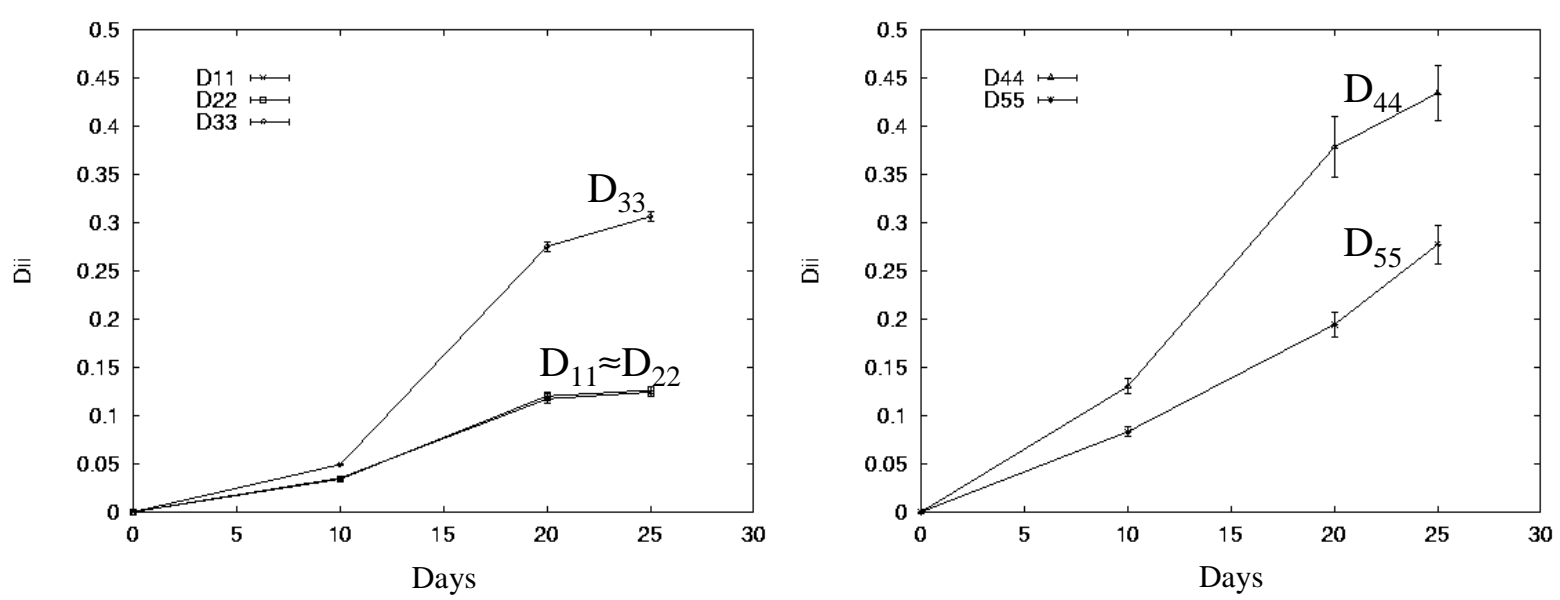

Figure 2. Damage evolution 


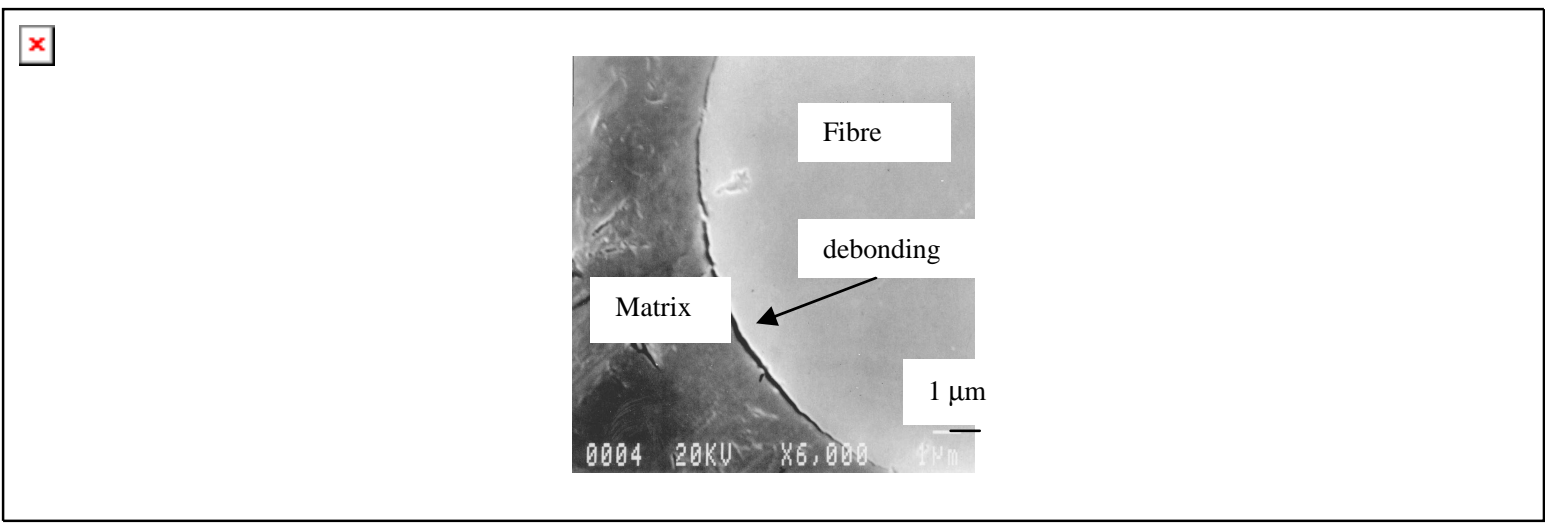

Figure. 3: SEM observation : fiber-matrix debonding

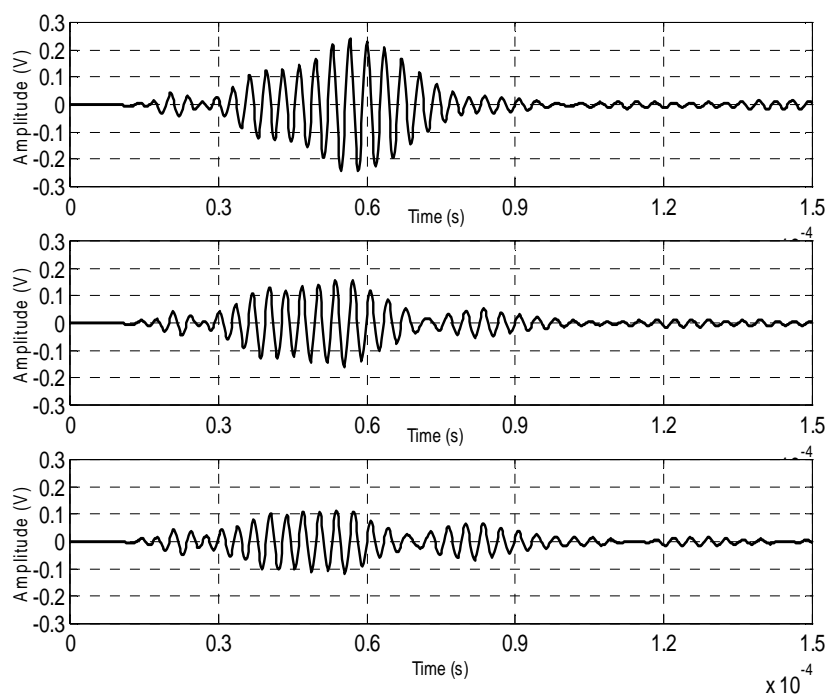

Figure. 4. Time signals transmitted between emitter and receiver in sane case (top), after the first (middle) and second 8-joules impact (bottom) 


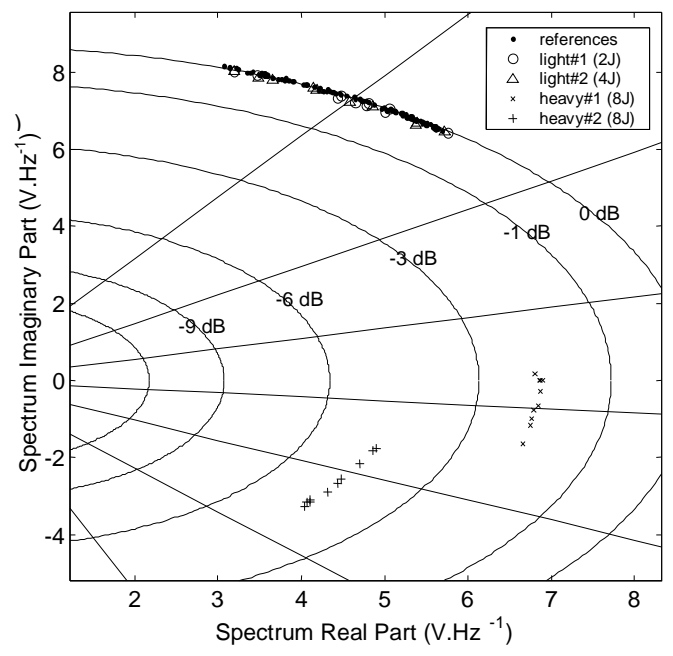

Figure. 5. Transmitted spectra maxima in CFRP plate for $\mathrm{S}_{0}$ mode.

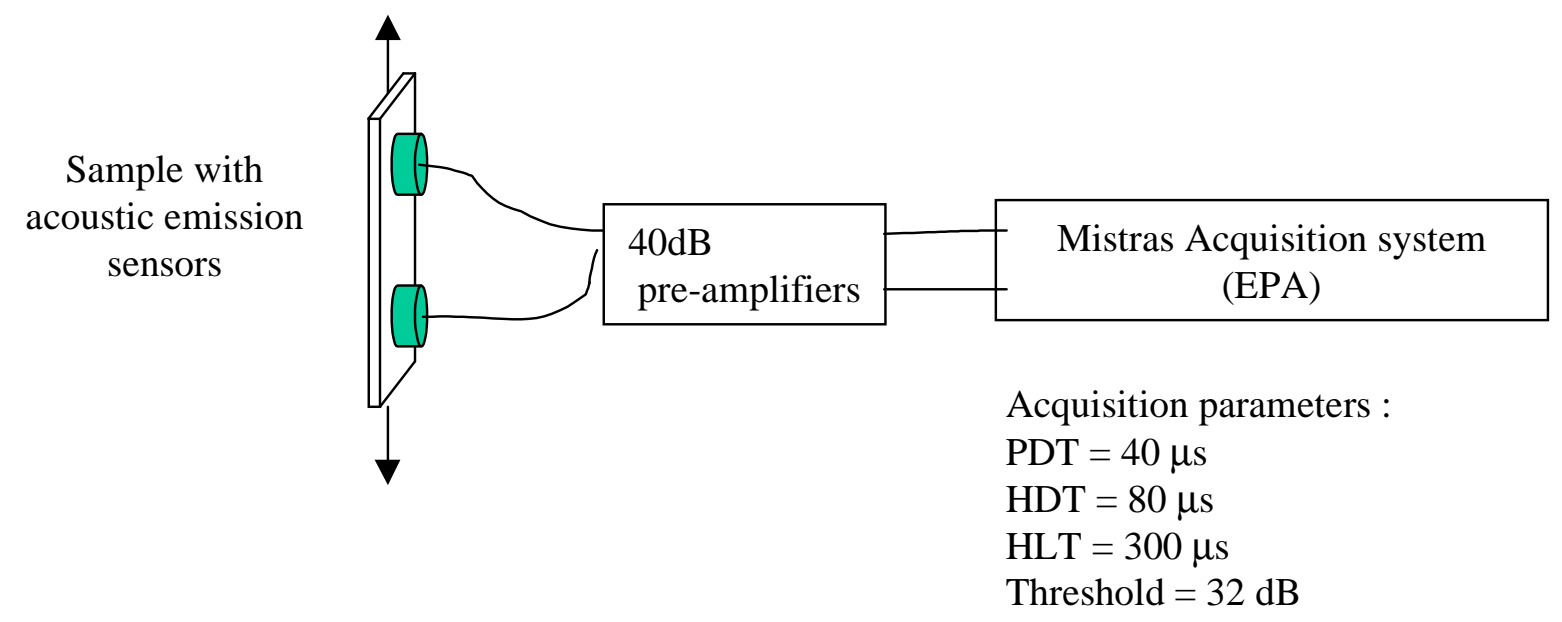

Figure $6:$ schema of tensile test with acoustic emission 


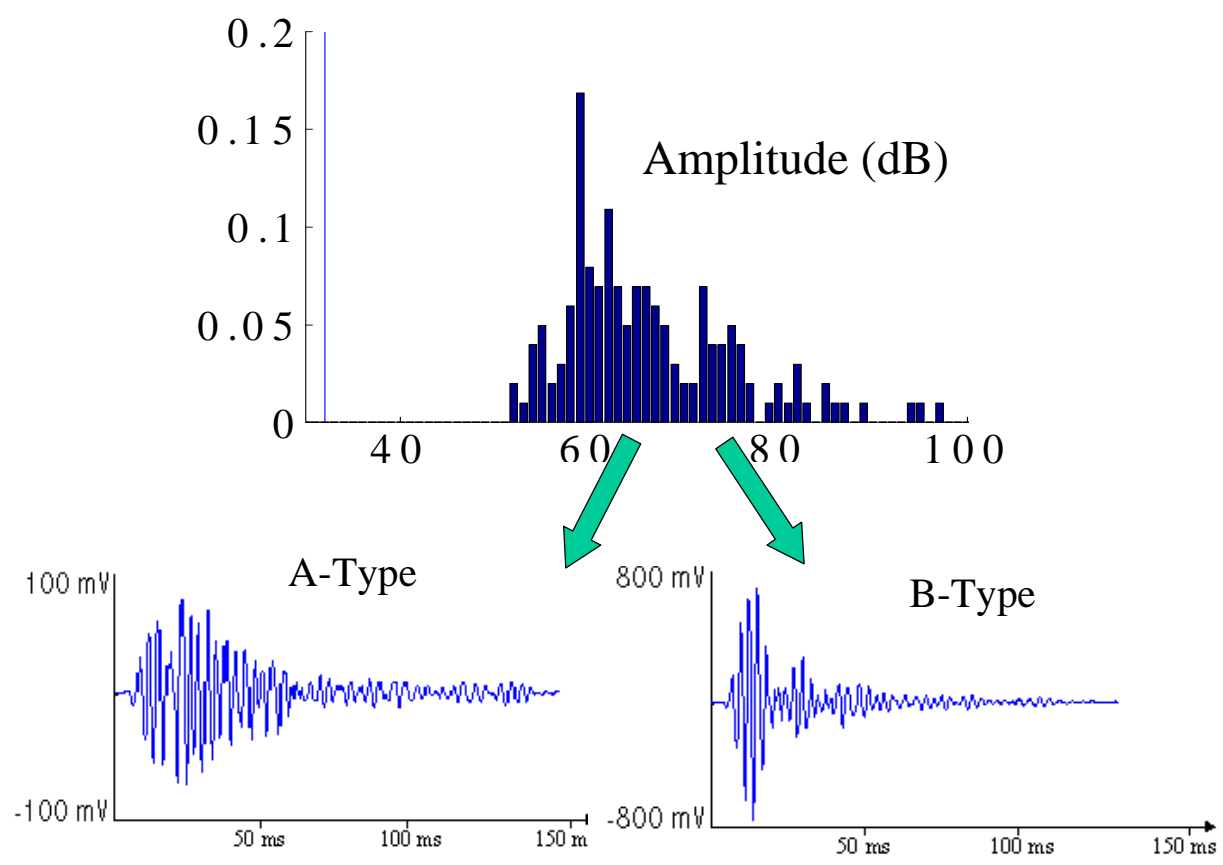

Figure 7 : amplitude distribution and typical waveforms of signals from tensile tests on $90^{\circ}$ samples

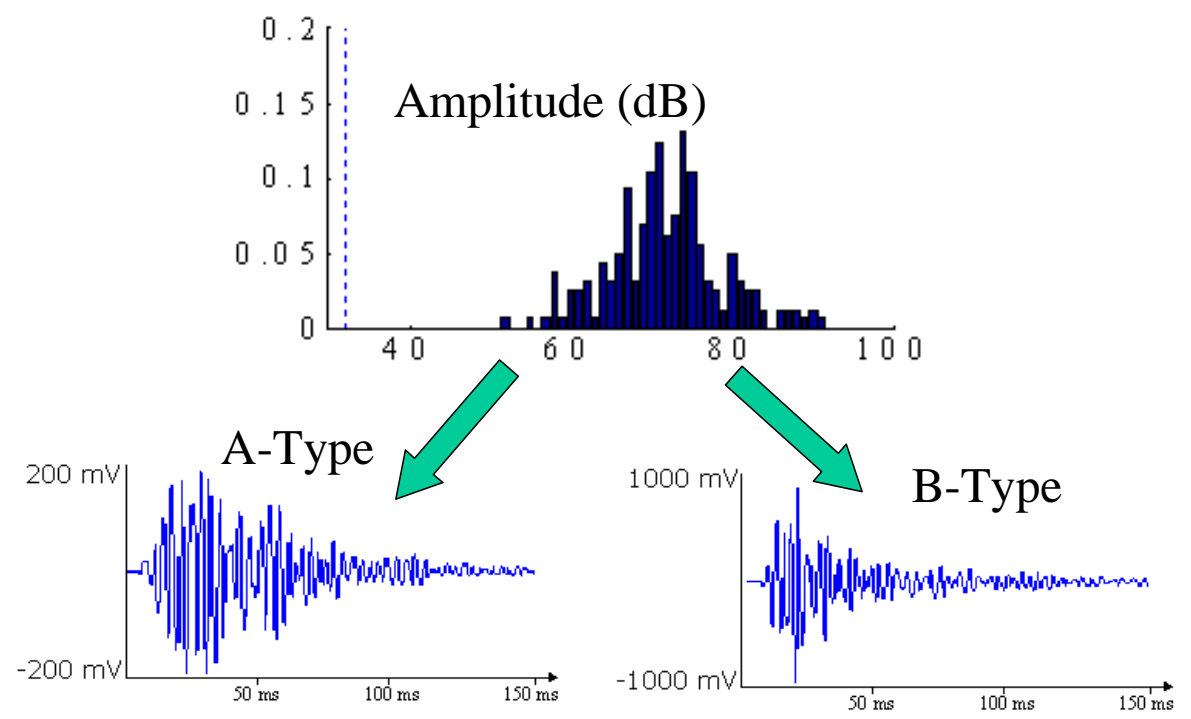

Figure 8 : amplitude distribution and typical waveforms of signals from tensile tests on $45^{\circ}$ samples 
Pure resin

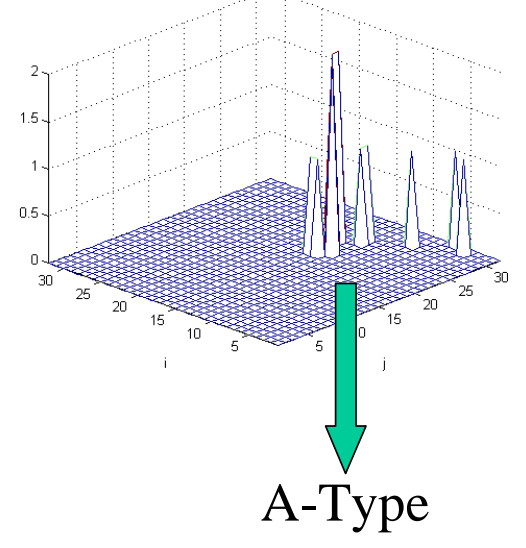

$90^{\circ}$

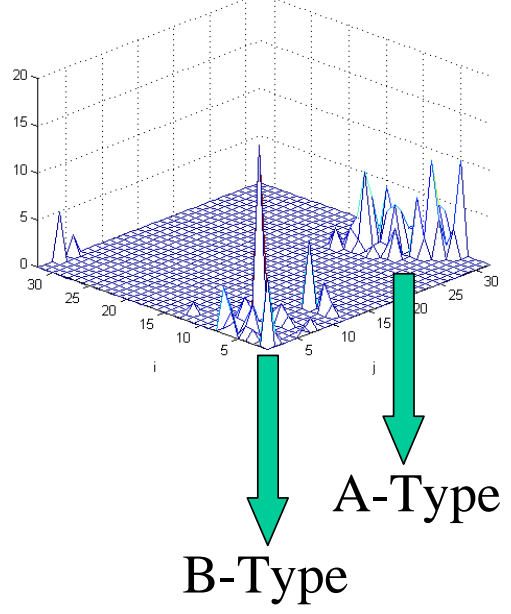

$45^{\circ}$

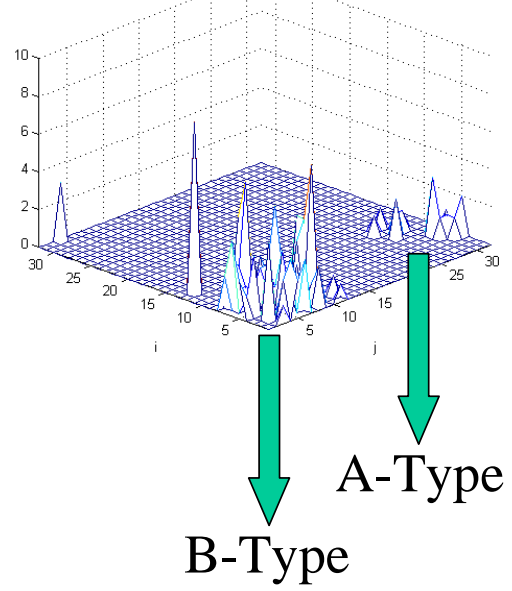

Figure 9 : activations of the Kohonen map for the three types of tensile tests
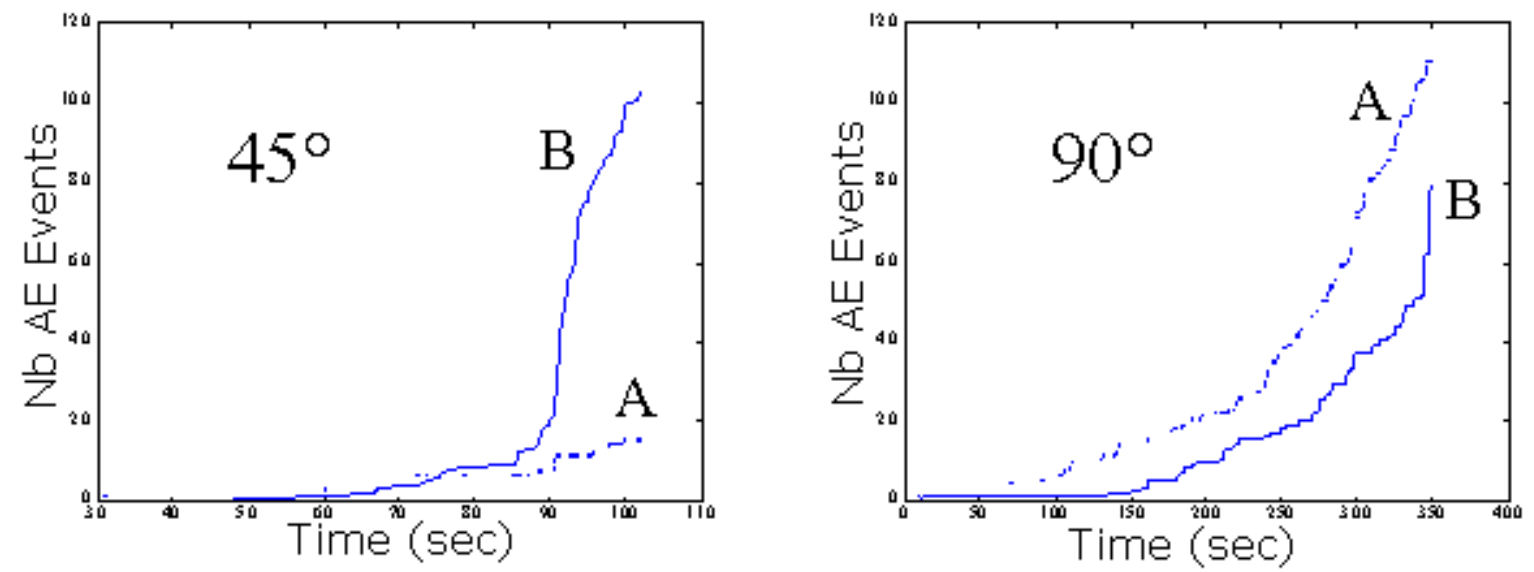

Figure 10 : AE activity evolutions of A-type and B-type during tests 